\title{
Students' use of symbolic forms when constructing differential length elements
}

\author{
Benjamin P. Schermerhorn, and John R. Thompson \\ Department of Physics and Astronomy and Maine Center for Research in STEM Education, \\ University of Maine, Orono, ME, 04469, USA
}

\begin{abstract}
As part of an effort to examine students' understanding of the structure of non-Cartesian coordinate systems and the differential vector elements associated with these systems, students in junior-level electricity and magnetism (E\&M) were interviewed in pairs. Students constructed differential length and volume elements for an unconventional spherical coordinate system. A symbolic forms analysis found that students invoked known as well as novel symbolic forms when building these vector expressions. Further analysis suggests that student difficulties were primarily conceptual rather than symbolic.
\end{abstract}

\section{INTRODUCTION}

Mathematics in physics is more than a mere calculation tool. Physics students use mathematics to reason in the analysis of physical systems when problem solving. The appropriate use of multivariable and vector calculus in upper-division E\&M involves interpreting physical situations, making determinations about coordinate systems, and constructing appropriate differential elements. In particular, these differential elements are often used as vectors when students construct integrals for Gauss's Law, Ampère's Law, and other vector calculus expressions. Students in E\&M have been shown to have particular difficulty setting up appropriate calculations, interpreting the results of calculations, and accounting for underlying spatial situations [1]. Thus in addition to being necessary to upper-division course work, mathematical understanding is a key area of difficulty for students.

The differential length element is of particular importance in E\&M. Not only are differential length elements used in setting up path integrals for finding electric potential difference and various quantities in magnetostatics, but understanding how these lengths are created (i.e., through changes in specific variables) is fundamental to determining the area and volume elements used when solving problems of flux and enclosed charge.

While previous work has addressed student use and understanding of integration [2-4], applications of Gauss's and Ampère's Laws [1, 6-8], and vector differential equations in mathematics and physics settings [9-10], little work has been done to address student understanding of differential elements and how they are constructed or determined in the non-Cartesian coordinate systems typically employed in E\&M. Pepper and colleagues identified an instance in a homework help session where students neglected to include the necessary scaling factors when writing spherical differential areas, using $d a=d \theta d \phi$ rather than $d a=r^{2} \sin \theta d \theta d \phi$ [1]. Another group of students attempted a line integral in three dimensions using $d z$ as a length element [1]. These errors are consistent with both a difficulty accounting for the underlying physical system and a limitation in students' understanding of how to construct and apply differential elements.

As part of a study on how students construct and make sense of differential vector elements in non-Cartesian coordinate systems, we designed an unconventional spherical coordinate system and asked students to build the differential length vector. The emphasis on equation construction at an interface between mathematics and physics understanding naturally lends itself to an analysis connecting students' internalized conceptual understanding with the structural aspects of this construction. Sherin's symbolic forms framework [11] was developed for this purpose. This paper describes the students' construction of differential length elements using Sherin's symbolic forms.

\section{SYMBOLIC FORMS}

Based on the knowledge-in-pieces model [12], a symbolic forms analysis guides investigations of students' thinking about equations. Sherin's work originated as an analysis of introductory students developing and discussing expressions to describe physical situations [11]. From this, the specific nature of a symbolic form arose as a combination of a conceptual schema - students' underlying ideas - and an intuitive symbol template - the specific structures of an equation. Symbol templates can represent clusters of terms in multiplication or addition as well as the dependence of a desired quantity on other specified variables. For example, $\square+\square+\square$ would appear in a students' equation if the student decided they needed to add multiple quantities. This particular symbolic form is known as parts-of-a-whole [11].

Meredith and Marrongelle adapted the conceptual side of symbolic forms to describe what cues students to integrate in electrostatics [4]. They identified dependence, the reliance on a particular quantity, and parts-of-a-whole as major cues for integration. Symbolic forms have been used to describe physical chemistry students' sense-making of partial derivatives in thermodynamics [13]. The ideas of symbolic forms were used to address students' conceptual schemata when making sense of various integrals, which was often mediated by a graphical representation [14]. 

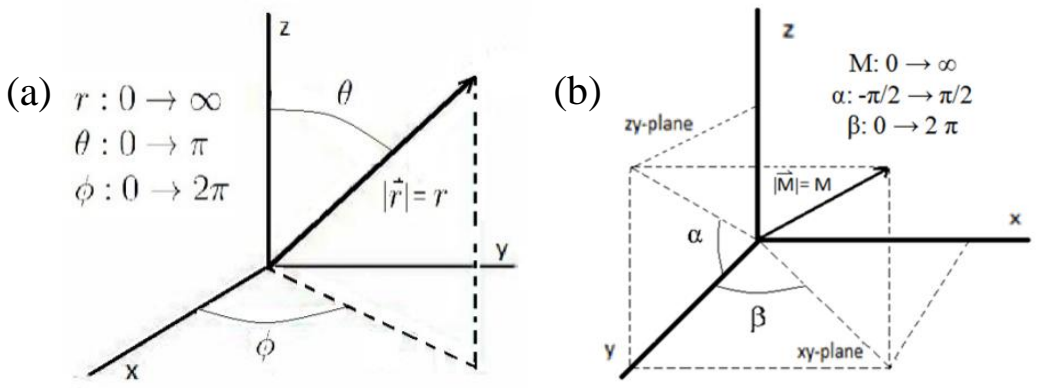

(c)

$$
\begin{aligned}
& \begin{aligned}
\overrightarrow{d l}=d r \hat{r}+r d \theta \hat{\theta}+r \sin \theta d \phi \hat{\phi} \\
d \tau=r^{2} \sin \theta d r d \theta d \phi
\end{aligned} \\
& \text { (d) } \begin{aligned}
\overrightarrow{d l} & =d M \hat{M}+M d \alpha \hat{\alpha}+M \cos \alpha d \beta \hat{\beta} \\
d \tau & =M^{2} \cos \alpha d M d \alpha d \beta
\end{aligned}
\end{aligned}
$$

FIG 1. (a) Conventional (physics) spherical coordinates; (b) an unconventional spherical coordinate system ("schmerical coordinates") given to students, for which they were to construct differential length and volume elements. The correct elements for each system are in (c) and (d), respectively.

\section{STUDY DESIGN \& METHODS}

In order to elicit students' understanding of how differential vector elements are created, we designed an unconventional spherical coordinate system, which we call schmerical coordinates (Fig. 1). This system is left-handed and depicts both angles as being measured from the $y$-axis. This affords us the ability to determine whether students are recalling the projection element from spherical coordinates or are recognizing that a $\cos \alpha$ is needed to adequately project into the plane of $\beta$.

Four clinical, think-aloud interviews were conducted with pairs of students $(\mathrm{N}=8)$ at the end of the first semester of a year-long, junior-level E\&M sequence. The use of pair interviews allowed for more authentic student-to-student interaction with minimal influence from the interviewer. Students were asked to conclude whether the given schmerical system was valid, thus fit for use when problem solving, and to construct and verify expressions for the differential line and volume elements.

Interviews were transcribed and analyzed first for patterns in the ideas used by groups as well as the sequence in which those ideas arose in the construction of the differentials [15]. These ideas include component and direction, dimensionality, differential, and projection.

Secondary analysis examined how students arranged terms in order to identify any common symbolic templates specific to the construction of vector differentials. We then sought to connect these templates to the conceptual discussions the students were engaged in regarding the writing of these expressions.

\section{RESULTS \& DISCUSSION}

Overall, no group was successful in their initial construction of the differential length element, due to inattention to certain ideas [15].

The first three subject pairs $(\mathrm{AB}, \mathrm{CD}$, and $\mathrm{EF})$ mostly discussed the need for the structure of the equation and the inclusion or exclusion of certain terms; the discussions of the fourth group $(\mathrm{GH})$ involve mainly recalled ideas. Given the emphasis of symbolic forms on the students' conceptual schemata, most of the evidence for symbolic form use comes from the first three interviews.

Analysis revealed invocation of several symbolic forms during the interviews (Table 1). Because the context involved both vector and differential quantities typically appearing in the upper division, we identified novel forms that would not have been accessible to previous studies focused on introductory content.

Analysis of the interview data shows evidence of students attending to the need for multiple components as well as the need for a change in each of the three spatial directions, consistent with Sherin's parts-of-a-whole form:

F: There are three different $d l$ 's. There is with respect to $M$, with respect to and with respect to $\beta$.

Group CD similarly began by recognizing the need to sum a term for each unit vector (Fig. 2). Students also used language associated with Sherin's no dependence symbolic form [11]:

A: For $\alpha$, it doesn't have any dependence on this other angle over here [sweeps arm down]...

Here A was reasoning about the geometrical space of the system and recognized that motion resulting from a change in $\alpha$ was independent of the other angle, $\beta$, whereas the $\hat{\beta}$ term includes a trigonometric function of $\alpha$.

In addition to invoking familiar symbolic forms, students also used new template-schema pairings. To acknowledge the vector nature of the differential length element, we identify a magnitude-direction symbolic form used by all student pairs. This form accounts for students recognizing the need to multiply a quantity, representing a magnitude, by a unit vector, which denotes the direction associated with that quantity. While this is similar to Sherin's scaling symbolic form [11], the magnitude here can have dimensions and/or units. As an example of the contribution of the magnitude-direction form, consider the vector $5 \mathrm{~m} \hat{\jmath}$, which is seen as a distance given in a specific 
Table I. Existing and novel symbolic forms identified in students' construction of a differential length element.

\begin{tabular}{lcl}
\hline \hline Symbolic Form & Symbol Template & Conceptual Schema \\
\hline Parts-of-a-whole & $\square+\square+\square$ & $\begin{array}{l}\text { Accounts for multiple components that contribute to a larger whole } \\
\text { (Sherin, 2001) } \\
\text { Indicates an expression is independent of, or not a function of, a specific } \\
\text { variable (Sherin, 2001) } \\
\text { No dependence }\end{array}$ \\
Coefficient & {$[\square \ldots]$} & $\begin{array}{l}\text { Represents a quantity seen as just a number or a constant (possibly } \\
\text { having units) put in front of an expression (Sherin, 2001) }\end{array}$ \\
Magnitude-direction & $\square \square$ & $\begin{array}{l}\text { Used to denote a vector expression including the magnitude of a quantity } \\
\text { (having units) and a unit vector to indicate a specific direction }\end{array}$ \\
Differential & $d \square$ & Represents taking a small amount of or infinitesimal change in a quantity \\
\hline \hline
\end{tabular}

direction. The scaling form is inappropriate, as $5 \mathrm{~m}$ is not seen as a number that attenuates the size of the unit vector. The coefficient form also has limited applicability in that the magnitude of a vector expression has specific meaning.

The invocation and combination of symbol templates occurred in a similar manner for each group (Fig. 3). At the beginning of construction, students immediately melded the templates for the magnitude-direction and parts-of-a-whole symbolic forms, accounting for the need to have a vector component in each of the three directions. C and D explicitly developed this structure by writing the three unit vectors associated with each direction while leaving space to fill in the magnitude of each component (Fig. 2).

As the magnitude term of each component part was broken down, students' discussion focused around how to incorporate a differential term, often with a preceding coefficient. From this we identify a differential symbolic form, which students would use to signify a small change or small amount of a given variable that in turn produces the differential length component. The template is similar to what appears in templates for integration [14] but here the differential is a standalone quantity with its own attached schemata. This form is also distinct from the change in the base \pm change symbolic form [11] in which the change is applied to a quantity or seen as a correction term dependent upon the base (i.e., $v_{o} \pm a t$ ).

As part of students' application of a conceptual schema, many students paired the variable in the differential to the unit vector (i.e., $d M \widehat{M}, d \alpha \hat{\alpha}$, and $d \beta \widehat{\beta}$ ). While groups $\mathrm{AB}$ and $\mathrm{GH}$ did this automatically, others attempted to forcefully insert a differential into the expression. Group $\mathrm{CD}$ wrote a " $d$ " in front of the whole expression $M \cos \alpha$ to get a differential (Fig. 4); student $F$ tried to identify an

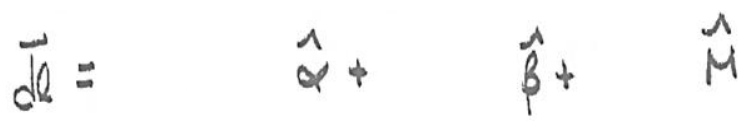

FIG 2. Beginning stages of construction for $\mathrm{C}$ and $\mathrm{D}$ showing the coupling of the parts-of-a-whole and magnitude-direction symbolic forms. infinitesimal arc length as $r \sin d \theta$. In fixing this, group EF focused their construction on the need for a differential length component in a particular direction to contain a differential with that variable, as seen in the excerpt below:

E: So you're going to have a length component in the $\beta$-hat direction...so, basically we're going to need...an $M$...so it's $M$ times some $\Delta$, I think it's $M$ times $\Delta \beta$, a small $\beta$, because it's like if you take $r$ times its small $\theta$ then that is the arc length.

EF articulated this as $\overrightarrow{d l}_{\beta}=M d \beta \hat{\beta}$, which still lacks the needed trigonometric projection term. Once the students connected the unit vector to the variable in the differential form, they correctly accounted for the larger pieces of the differential length vector and focused their efforts on determining the coefficients for the differentials.

As students made determinations of the coefficients of the angle components, they often used Sherin's coefficient form to discuss the inclusion of specific terms. The determination of the coefficients of each term occurred at various stages of construction and for several reasons, including recall and mapping to spherical coordinates to recognizing $M$ as the radius in an arc-length. The need for an arc length was attached to a geometric conceptual schema in which students recognized that the needed length for an angle component was along the small arc associated

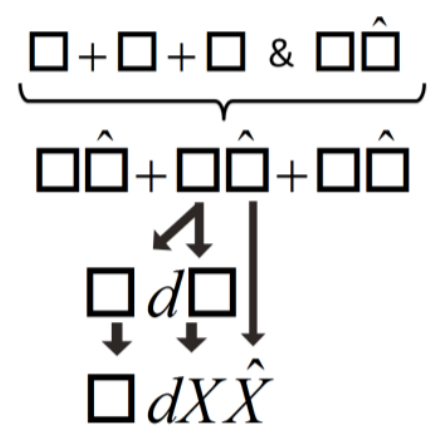

FIG 3. The general flow of students' use and combination of symbol templates during the interview task. 


$$
\overrightarrow{d l}=\stackrel{M \sin (\alpha)}{\hat{\alpha}+}+\frac{d \cos (\alpha)}{\beta}+d M \hat{M}
$$

FIG 4. Students $C$ and D incorrectly incorporating the idea of a differential.

with a small change in the angle. In many cases, the predominant reason for including $M$ in a component was dimensionality as seen in these two (independent) excerpts:

A: ...This doesn't have any units of length, so it needs to have some $M$ term.

C: ....So, if it's going to be some trig thing but sine of something isn't a length so we're going to have to also have something else in there.

We identify these as abstracted uses of the dependence symbolic form [11], where the dependence is not on a specific variable but instead is driven by dimensional analysis. The $\cos \alpha$ term was generally absent from students' coefficients, suggesting that students' conceptual schemata failed to account for the projection of $M$ into the $\beta$ plane. This explains the reliance on recall or the decision to not include a trigonometric function at all.

\section{CONCLUSIONS}

A symbolic forms analysis enables us to see what students think a vector differential length element should look like as well as what concepts they use to construct these elements. This analysis in an upper-division context has led to the identification of new symbolic forms arising from the increased mathematical sophistication as well as a sequence of invocation of these forms during construction.

While Sherin found some students struggling to invoke the correct symbol template, our students were able to recognize the general form or structure needed for the equation and ended up with the correct symbol template. Difficulties with the construction of expressions seem to be related almost exclusively to the conceptual schema, e.g., neglecting the role of projection. This suggests that any instructional reform needs to target the conceptual ideas that are associated with the building of the differential length element, specifically with regard to the determination of scaling factors.

Attention to dimensionality and units played a key role in students' construction of vector differential expressions, but some students struggled to assign correct dimensions to certain terms. This is a significant finding, as prior research on symbolic forms at the introductory level effectively neglected students' choice of units in the analysis [11].

Additionally, geometric reasoning was prominent in students' conceptual schemata, specifically in visualizing the lengths associated with small changes in individual variables. When constructing vector differential lengths, students attended to the need for multiple components and connected differentials to unit vectors of the same variable. In cases where students had difficulty, recall mediated the construction of their expressions, as seen with partial derivative expressions [13]. In our study, however, recall often led students to incorrect expressions (e.g., both groups $\mathrm{AB}$ and $\mathrm{CD}$ included a sin $\alpha$ term [15]).

Later in these interviews, students constructed and checked the differential volume element; this led students to correct their individual length elements. These findings will inform the development of instructional resources for E\&M and/or math methods courses. Further efforts will explore student reasoning about differential area elements and student use of specific coordinate systems in these contexts.

\section{ACKNOWLEDGEMENTS}

The authors would like to acknowledge the University of Maine Physics Education Research Laboratory, as well as collaborators Michael Loverude, Joseph Wagner, and Warren Christensen, for input into the development of the project and insight on interpretation of results. This material is based upon work supported by NSF grant PHY-1405726.
[1] R. Pepper, S. Chasteen, S. Pollock, and K. Perkins, Phys. Rev. ST - Phys. Educ. Res., 8, 010111 (2012).

[2] L. Doughty, E. McLoughlin, and P. van Kampen, Am. J. Phys., 82, 1093-1103 (2014).

[3] D. Hu and N. S. Rebello, Phys. Rev. ST - Phys. Educ. Res., 9, 020108 (2013).

[4] D. Meredith and K. Marrongelle, Am. J. Phys., 76, 570-578 (2008).

[5] D. Nguyen and N. S. Rebello, Phys. Rev. ST - Phys. Educ. Res., 7, 010113 (2011).

[6] J. Guisasola, J. Almudí, J. Salinas, K. Zuza, and M. Ceberio, Eur. J. Phys., 29, 1005-1016 (2008).

[7] C. Manogue, K. Browne, T. Dray, and B. Edwards, Am. J. Phys., 74, 344-350 (2006).
[8] C. Wallace and S. Chasteen, Phys. Rev. ST - Phys. Educ. Res., 6, 020115 (2010).

[9] C. Astolfi and C. Baily, 2014 Phys. Educ. Res. Conf. AIP CP, 11, 31-34 (2014).

[10] L. Bollen, P. van Kampen, and M. De Cock, Phys. Rev. ST - Phys. Educ. Res., 11, 020129 (2015).

[11] B. Sherin, Cogn. Instr., 19, 479-541 (2001).

[12] A. diSessa, Cogn. Instr., 10, 105-225 (1993).

[13] N. Becker and M. Towns, Chem. Educ. Res. Pr., 13, 209-220 (2012).

[14] S. Jones, J. Math. Behav. 32, 122-141 (2013).

[15] B. Schermerhorn and J. Thompson, in Proc. $19^{\text {th }}$ Ann. Conf. on Res. in Undergraduate Math. Educ. (MAA, 2016, accepted). 